\title{
Surgical Aspirator with Steam-Jet Coagulator for Hepatic Surgery
}

\author{
Hitoshi Yoshiki ${ }^{1}$, Kotaro Tadano ${ }^{1}$, Kenji Kawashima ${ }^{2}$ \\ ${ }^{1}$ Tokyo Institute of Technology \\ 4259 R2-46 Nagatsuta-cho, Midori-ku, Yokohama, Japan \\ yoshiki.h.aa@m.titech.ac.jp; tadano@pi.titech.ac.jp \\ ${ }^{2}$ Tokyo Medical and Dental University \\ 1-5-45 Yushima, Bunkyo-ku, Tokyo, Japan \\ kkawa.bmc@tmd.ac.jp
}

\section{Extended Abstract}

Hepatectomy is a radical treatment for hepatic neoplasms, both benign and malignant. The most basic liver resection method is that surgeon crushes liver parenchyma with basic surgical clamps (so-called "crush-clamp" technique) [1]. The crush-clamp method generates bleeding cross-sections of livers, and thus surgeons perform the Pringle maneuver and hemostasis with several cauterization devices. The Pringle maneuver is usually performed to reduce blood loss, and to maintain coagulation function of surgical energy devices. The maneuver is a technique to minimize blood loss during hepatic surgery by pinching the vascular pedicle. The technique consists of 15-30 minutes clamping and 5-30 minutes reperfusion in order to avoid damages and complications [2,3]. This repetitive reperfusions of the maneuver consume times of medical practitioners performing a hepatic surgery. Vessel sealing systems or vascular clips are used for hemostasis of larger vessels while monopolar electrocautery devices or argon plasma coagulators are used for stop bleeding from small vessels at cross-sectional surface of the liver parenchyma. However, monopolar devices are difficult to perform coagulation effectively with bleeding surface, and argon plasma coagulators have fatal risks of gas embolism caused by argon gas [4].

To realize a device which can perform cauterization of bleeding parenchyma without fatal complications, we propose the surgical aspirator with steam-jet coagulator (SASCo). The SASCo performs quick surficial cauterization with steamjets and blood removal by suctioning and by the jet effect. The quick blood removal and cauterization on bleeding surface let surgeons reduce the operation time, the damage to remained liver, and the risks of complications.

In this study, we prototyped the SASCo, and evaluated the feasibility of the proposed method through in-vivo cauterization experiments and morphological analysis.

The ex-vivo evaluation showed the effectiveness of the integration of the steam-jet coagulation and the suction for reduction of collateral thermal damage. The cauterization of bleeding porcine liver with the SASCo prototype were successfully performed in the in-vivo study. Moreover, two different types of working state (a vacuuming coagulation and a contactless coagulation) were observed. The morphological analysis showed characteristics of cauterization of both vacuuming and contactless coagulations. These results indicate the effectiveness and the feasibility of the SASCo as a surgical cauterization device for hepatic surgeries.

\section{References}

[1] R. J. Aragon and N. L. Solomon, "Techniques of hepatic resection,” J. Gastrointest. Oncol., vol. 3, no. 1, pp. 28-40, 2012.

[2] K. Man, S. T. Fan, I. O. Ng., et al., "Prospective evaluation of Pringle maneuver in hepatectomy for liver tumors by a randomized study," Ann. Surg., vol. 226, no. 6, pp. 704-13, 1991.

[3] S. A. Dello, K. W. Reisinger, R. M. van Dam, et al., "Total intermittent Pringle maneuver during liver resection can induce intestinal epithelial cell damage and endotoxemia," PLOS ONE, vol. 7, no. 1, pp. e30539, 2012.

[4] G. Sankaranarayanan, R. R. Resapu, D. B. Jones, et al., "Common uses and cited complications of energy in surgery," Surg. Endosc., vol. 27, no. 9, pp. 3056-72, 2013. 
[5] H. Yoshiki, K. Tadano, D. Ban, et al., "Development of contactless cauterization device for surgery using a steamjet," J. Biomed. Eng. Med. Imag., vol. 3, no. 6, pp. 1-12, 2016. 